\title{
Coherent and incoherent transport through T-shaped double quantum dots
}

\author{
V. Moldoveanu ${ }^{\mathrm{a}}$, M. Ţolea ${ }^{\mathrm{a}}$, B. Tanatar ${ }^{\mathrm{b}, *}$ \\ ${ }^{a}$ National Institute of Materials Physics, P.O. Box MG-7, Bucharest-Magurele, Romania \\ ${ }^{\mathrm{b}}$ Department of Physics, Bilkent University, Bilkent, 06800 Ankara, Turkey
}

Available online 24 August 2007

\begin{abstract}
We investigate the measurement induced dephasing of the Fano effect in the electronic transport through a double quantum dot mesoscopic interferometer coupled to a charge detector. The current and the differential conductance are computed within the Keldysh formalism, taking into account of the inelastic processes due to the dot-detector interaction. We show that the visibility of the Fano lineshape is reduced by applying a finite bias on the charge detector.
\end{abstract}

(C) 2007 Elsevier B.V. All rights reserved.

PACS: $73.23-\mathrm{b} ; 73.63 . \mathrm{Kv}$

Keywords: Coherent quantum transport and dephasing

\section{Introduction and model}

The decoherence of quantum transport in mesoscopic interferometers is a timely issue both from experimental and theoretical points of view, particular attention being paid to the loss of interference when reading 'Which Path-type information [1]. It was shown that the Aharonov-Bohm oscillations of the current through a single dot interferometer are reduced when a charge detector subjected to a finite bias is placed in the vicinity of the embedded dot. This effect is due to the inelastic scattering processes induced by the dot-detector Coulomb interaction and was discussed theoretically and reproduced qualitatively in Ref. [2]. Another mesoscopic interferometer which shows interesting properties is the so-called $\mathrm{T}$-shaped system, or the side-coupled dot. In the experiment of Kobayashi et al. [3] it was shown that the current exhibits Fano lineshapes as a function of the gate potential applied on the side-coupled dot. Also, Johnson et al. [4] emphasized the interplay of Coulomb interaction and Fano

*Corresponding author. Tel.: + 903122901591 ; fax: + 903122664579.

E-mail address: tanatar@fen.bilkent.edu.tr (B. Tanatar). interference in quantum dots coupled to a one-dimensional channel.

In this paper we present related results for a different system, namely a double quantum dot coupled to a charge detector. One of the dots is connected to leads and provides the background contribution to the transport. The second one is only side-coupled and interacts with a nearby charge detector. We consider single site quantum dots. The Hamiltonian of the system (interferometer + detector) reads as

$H=\sum_{i=1}^{2}\left(\varepsilon_{i}+V_{i}\right) d_{i}^{\dagger} d_{i}+\tau\left(d_{1}^{\dagger} d_{2}+\right.$ h.c $)+\varepsilon_{0} a^{\dagger} a+U d_{2}^{\dagger} d_{2} a^{\dagger} a$,

where $d_{i}^{\dagger}\left(d_{i}\right)$ are creation (annihilation) operators on the two dots, $a^{\dagger}(a)$ are operators associated with the detector and $\varepsilon_{i}, \varepsilon_{0}$ are on-site energies. $\tau$ is the hopping constant between the dots and $U$ is the strength of the Coulomb interaction between the second dot and the detector. $V_{i}$ is a plunger gate voltage applied on the $i$ th dot. In the steady state regime the standard application of the Keldysh 
a

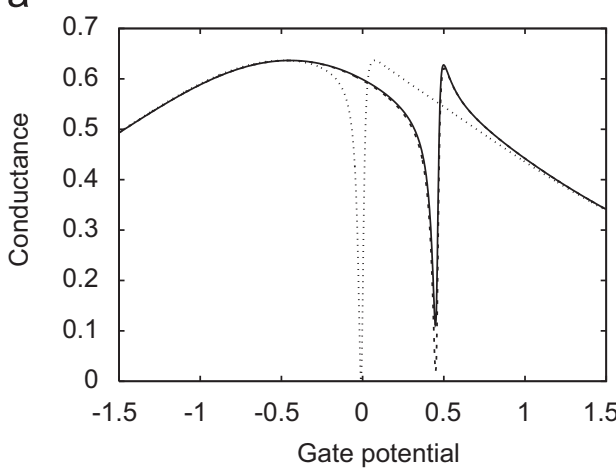

C

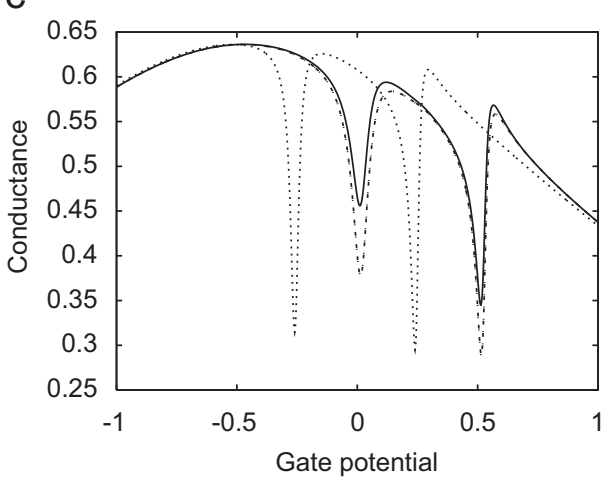

b

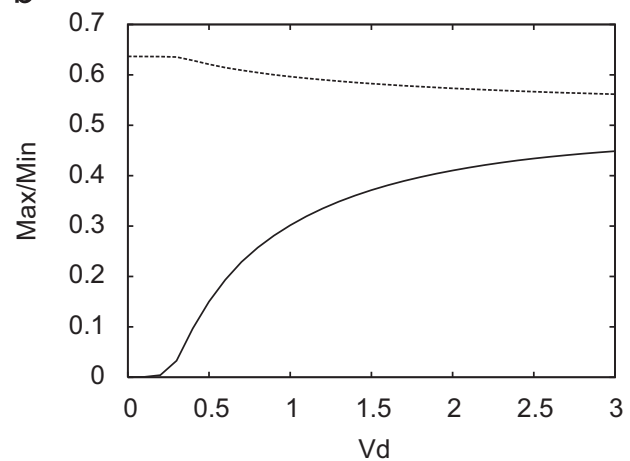

d

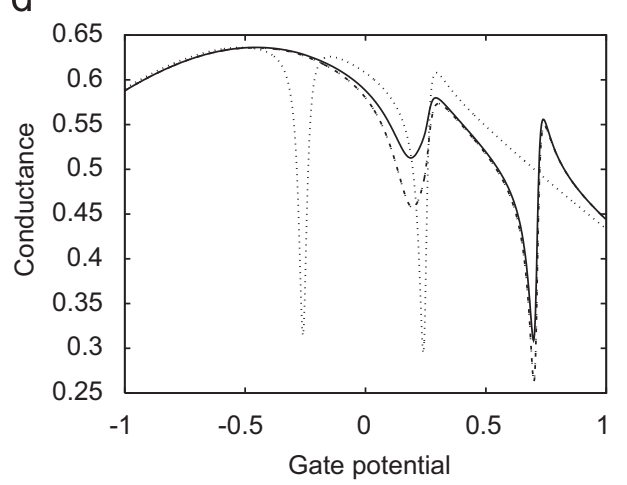

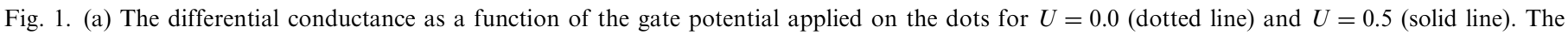

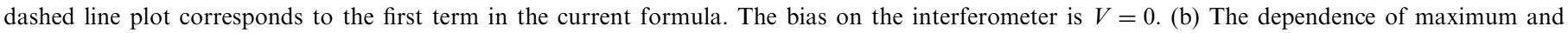

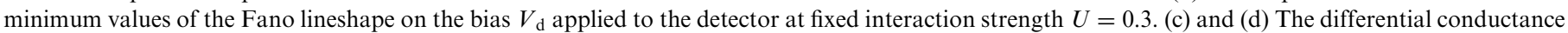

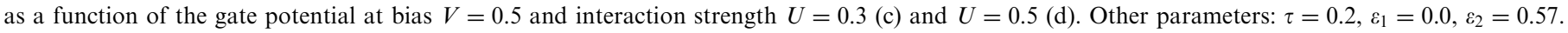

machinery and current conservation lead to the following formula for the current:

$J=\frac{e}{h} \int_{-2 t_{\mathrm{L}}}^{2 t_{\mathrm{L}}} \mathrm{d} E\left(\Gamma^{2}\left|G_{11}^{\mathrm{R}}\right|^{2}-\Gamma G_{21}^{\mathrm{R}} \operatorname{Im} \sum_{22}^{\mathrm{R}} G_{21}^{\mathrm{A}}\right)\left(f_{\alpha}-f_{\beta}\right)$,

where $\Gamma=2 \pi t_{\mathrm{L}}^{2} \rho$ in which $\rho=\sqrt{4 t_{\mathrm{L}}^{2}-E^{2}} /\left(2 \pi t_{\mathrm{L}}^{2}\right)$ is the density of states in the lead and $t_{\mathrm{L}}$ is the hopping energy. Note that we do not use the wide-band limit approximation and therefore the density of states depends on energy. $\Sigma^{\mathrm{R}}$ is the interaction self-energy that will be computed perturbatively up to the second order in the interaction strength (for more details see Ref. [2]). We also remark that the Green functions contain the self-energy of the leads which we denote by $\Sigma_{\mathrm{L}}$ as well as the interaction self-energy $\Sigma^{\mathrm{R}}$. The above equation clearly shows that as long as the interaction self-energy has a non-vanishing imaginary part the current cannot be cast in a Landauer-Büttiker formula. This fact was emphasized at a general level by Meir and Wingreen [5] and more recently König and Gefen [6] pointed out that for a single site dot the correct transmission coefficient in the presence of electron-electron interaction is $T(E)=$ $-\Gamma \operatorname{Im} G_{11}^{\mathrm{R}}$ instead of the Landauer form $T(E)=\Gamma^{2}\left|G^{\mathrm{R}}\right|$. For our system it is very easy to see that using the identity $\operatorname{Im} G_{11}^{\mathrm{R}}=\left[G^{\mathrm{R}}\left(\Sigma^{\mathrm{R}}+\Sigma_{\mathrm{L}}^{\mathrm{R}}\right) G^{\mathrm{A}}\right]_{11}$ the current formula can be rewritten as

$J=-e / h \int_{-2 t_{\mathrm{L}}}^{2 t_{\mathrm{L}}} \mathrm{d} E \Gamma(E) \operatorname{Im} G_{11}^{\mathrm{R}}(E)\left(f_{\alpha}(E)-f_{\beta}(E)\right)$.

When computing the differential conductance $G_{\mathrm{d}}(V)=$ $\mathrm{d} I(V) / \mathrm{d} V$ it is enough to take the derivative of $f_{\alpha}-f_{\beta}$ only since it turns out that $\operatorname{Im} \sum_{i, 22}^{\mathrm{R}}$ has a very weak dependence on the bias $V$.

\section{Results}

We present now the numerical results. The bias on the interferometer and on the detector are introduced by taking different chemical potentials of the leads. For example the bias on the interferometer $V=\mu_{\mathrm{L}}-\mu_{\mathrm{R}}$. In Fig. 1a we show the differential conductance for two values of the interaction strength $(U=0.0$ and 0.5$)$ as a function of the gate potential applied on the dots. As expected, $G_{\mathrm{d}}$ is a mixture of a broad resonance originating from the $Q D_{1}$ which is strongly coupled to the leads (i.e. $t_{\mathrm{L}}=1$ ) and a sharp resonance due to the level of the second dot whose imaginary part is of order $\tau^{2}$. The resulting Fano lineshape is shifted and reduced as the interaction strength increases. The current through the detector in turn exhibits two steps, the passage between them corresponding to the Fano 
resonance. For the interacting case we plot (the dashed line curve in Fig. 1a) the contribution of the first term in the current formula which is of a Landauer-Büttiker form, still containing the interaction effect. This term alone would lead to dephasing but the Fano dip increases more when the second term is taken into account. The fact that the Fano dip is not strictly zero is due to the non-vanishing imaginary part of the interaction self-energy. It is easy to show that the numerator of the effective Green function of the double dot behaves like $\varepsilon_{2}-\Sigma^{\mathrm{R}}(E)-E$. From the physical point of view the reduction of the Fano dip means that the Coulomb interaction suppresses the destructive interference. The same effect was discussed by König and Gefen [6].

Fig. 1b demonstrates the enhancement of dephasing when the bias on the detector increases. While keeping $U=$ 0.3 we give the Fano peak and dip values as functions of $V_{\mathrm{d}}$. The amplitude of the Fano line is drastically reduced (by $80 \%$ ). Note that the dependence on $V_{\mathrm{d}}$ is nonlinear and that the dephasing saturates at large values of $V_{\mathrm{d}}$. The saturation appears when the efficiency of the detector reaches its maximum.

In Figs. 1c and d we present the differential conductance when the bias on the interferometer is $V=0.5$. The main difference when comparing to Fig. 1a is that the conductance exhibits two dips. This is due to the fact that at finite bias the derivative of the Fermi functions lead (in the zero temperature limit) to two delta-functions $\delta(E \pm V / 2)$. The dotted line in Figs. 1c and d is the noninteracting conductance and it is clear that the two dips are located at 0.25 and -0.25 , which are up to a sign the chemical potentials of the two leads. Again, the interaction effect is to shift the resonances and to reduce the Fano dips.

Given the experiment of Kobayashi et al. [3] with sidecoupled dots and the high tunability of double quantum dots we hope our result will stimulate a similar setup with a charge detector. We mention also a recent paper by Malyshev et al. [7] on non-interacting side-coupled double dots which presents the time evolution of an incoming narrow Gaussian packet. The theoretical formulation we have previously developed [2] and briefly discussed here should be applicable to this and similar systems.

\section{Acknowledgements}

B.T. is supported by TUBITAK (106T052) and TUBA. V.M. is supported by TUBITAK-BIDEB and CEEX-2976.

\section{References}

[1] E. Buks, R. Schuster, M. Heiblum, D. Mahalu, V. Umansky, Nature (London) 391 (1998) 871.

[2] V. Moldoveanu, M. Tolea, B. Tanatar, Phys. Rev. B 75 (2007) 045309.

[3] K. Kobayashi, H. Aikawa, A. Sano, S. Katsumoto, Y. Iye, Phys. Rev. B 70 (2004) 035319.

[4] A.C. Johnson, C.M. Marcus, M.P. Hanson, A.C. Gossard, Phys. Rev. Lett. 93 (2004) 106803.

[5] Y. Meir, N. Wingreen, Phys. Rev. Lett. 68 (1992) 2512.

[6] J. König, Y. Gefen, Phys. Rev. Lett. 86 (2001) 3855.

[7] A.V. Malyshev, P.A. Orellana, F. Domnguez-Adame, Phys. Rev. B 74 (2006) 033308. 